\title{
La planification familiale postpartum au Burkina Faso - résumé en français
}

STEP UP Research Programme Consortium

Follow this and additional works at: https://knowledgecommons.popcouncil.org/departments_sbsr-rh

Part of the Demography, Population, and Ecology Commons, Family, Life Course, and Society Commons, International Public Health Commons, Maternal and Child Health Commons, Obstetrics and Gynecology Commons, and the Women's Health Commons How does access to this work benefit you? Let us know!

\section{Recommended Citation}

"La planification familiale postpartum au Burkina Faso - résumé en français." London: London School of Hygiene and Tropical Medicine, 2014. 


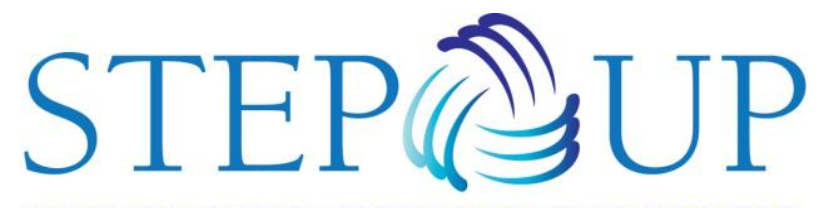

STRENGTHENING EVIDENCE FOR PROGRAMMING ON UNINTENDED PREGNANCY

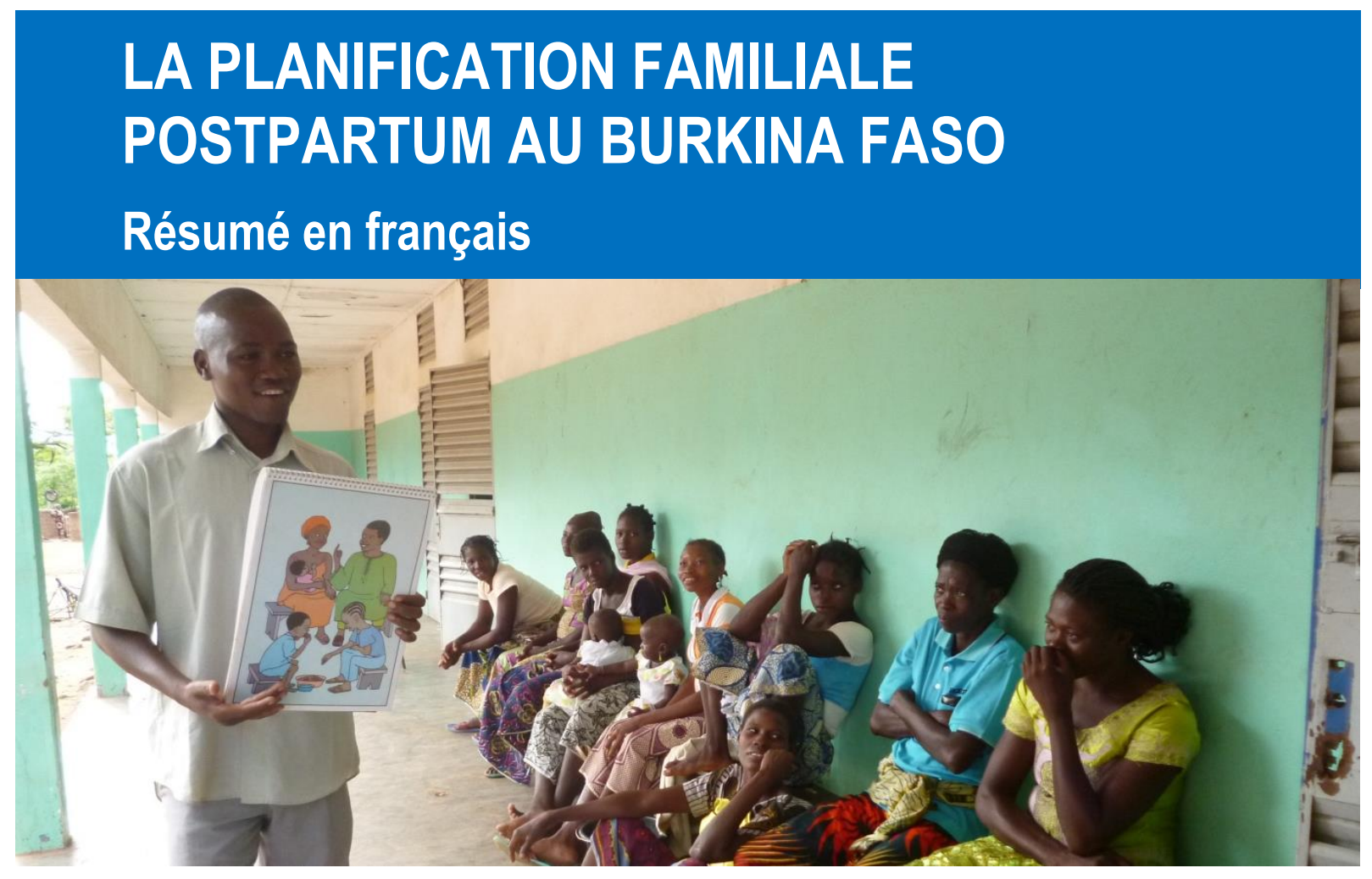

Objectifs de l'étude:

1. Donner un aperçu de la disponibilité et la qualité des services prénatals, du post-partum (PP) et de planification familiale (PF) au niveau des soins primaires, et évaluer la mesure dans laquelle ils fournissent des services efficaces de planification familiale postpartum (PFPP)

2. Evaluer l'accessibilité des services PFPP

3. Explorer l'effet de facteurs sociaux , économiques et culturels sur la demande et l'adoption de la PFPP

4. Estimer l'impact, la pertinence et la mise en œuvre de la politique nationale et des protocoles cliniques par rapport à la PFPP

5. Identifier les principaux obstacles qui empêchent une réponse efficace aux besoins non satisfaits en matière de PPFP aux niveaux de l'offre, de l'accès et de la demande, et proposer des domaines d'amélioration potentiels.

\section{Méthodologie:}

Un ensemble de trois méthodes qualitatives a été utilisée: une revue de la littérature et des politiques et documents clés, 20 entretiens semi -structurés avec usagers et d'autres parties prenantes, y compris les prestataires de soins, et 12 jours d'observation des interactions client - prestataire dans 4 CSPS (2 en milieu urbain, 2 en milieu rural). Le travail de terrain a été réalisée lors d'une visite de 5 semaines au Burkina en été 2013. Les observations dans les CSPS ont été réalisées dans le District de Dafra (BoboDioulasso). 


\section{Sommaire des résultats:}

Du point de vue de l'offre (Objectif 1), cette étude révèle d'importantes lacunes dans la disponibilité des services de PFPP de qualité dans les centres de soins primaires. Aucune discussion sur la préparation au post-partum, l'allaitement ou la PF a lieu lors des consultations prénatales individuelles. Dans les consultations post-partum, la qualité du counseling en PF n'est pas fiable et semble dépendre en grande partie du prestataire individuel. La PF n'est pas toujours mentionné lors des consultations de la sixième heure et du sixième jour. Ainsi, même si la contraception est offerte à la sixième semaine, nombreuses femmes ne sont pas prêtes à adopter une méthode: elles sont soit pas au courant qu'elles auraient dû faire un choix, soit elles n'ont pas été correctement conseillées lors tout contact précédent, ou bien elles n'ont pas apporté assez d'argent. Quel que soit le moment dans lequel les consultations ont lieu, les prestataires ne prennent généralement pas en compte l'histoire contraceptive de la femme ou les circonstances de sa vie, afin d'encourager le choix de la méthode la plus approprié et d'identifier le moment optimal pour son adoption. Les femmes ne sont pas toujours informées de l'ensemble des méthodes disponibles ou des effets secondaires, en partie en raison des préjugés des prestataires contre certaines méthodes, et aucun effort n'est fait pour encourager les hommes à participer aux consultations. L'attitude des prestataires en ce qui concerne la possibilité d'informer adéquatement les femmes et la participation des hommes est ambivalente et largement découragé. L'éventail des méthodes actuellement disponibles est limité, en raison des facteurs suivants: ruptures de stock, réticence des prestataires à faire connaître et fournir certaines méthodes, et manque de compétences. II faut ajouter aussi le fait que la politique actuelle en matière de santé de la reproduction empêche aux accoucheuses de fournir les méthodes contraceptives réversibles à longue durée d'action comme l'implant et le DIU.

En ce qui concerne l'accès (Objectif 2), les femmes font face à des obstacles géographiques et ainsi les rendez-vous de routine à 6 jours et 6 semaines après l'accouchement sont peu fréquentés. Les transferts pour obtenir une méthode qui n'est pas disponible localement sont très difficiles. Le coût des produits contraceptifs reste aussi une barrière significative (par exemple, le DIU coûte 800 CFA et l'implant 1000 CFA). La politique d'exemption pour d'autres consommables (pour l'insertion et le retrait de l'implant et du DIU) n'est pas pleinement appliquée. Malgré tout, d'autres occasions dans le continuum des soins sont gaspillées au cours lesquelles on pourrait fournir des conseils sur la PF, y compris la consultation prénatale (CPN). Les femmes aménorrhéiques qui exigent une méthode contraceptive plus de six semaines après l'accouchement rencontrent des obstacles spécifiques, tels que le prix du test de grossesse (entre 650 et 1500 CFA), ou l'exigence de stimuler une hémorragie de privation.

La demande et l'adoption de la PF (Objectif 3) sont généralement faibles pendant le postpartum. Bien que l'espacement des naissances soit estimé, il est possible que le désir d'une famille nombreuse joue un rôle dans la détermination de la faible demande. En outre, la persistance de coutumes spécifiques et les normes sociales telles que la séparation des époux et l'abstinence pendant le post-partum ont encore un impact sur l'adoption de la contraception, en particulier dans les zones rurales. L'opposition des hommes à la PF apparaît comme un thème fort. Le manque d'informations et les rumeurs contribuent également à donner à la contraception moderne une mauvaise réputation.

Des obstacles importants existent au niveau des politiques sur la PFPP (Objectif 4), surtout en ce qui concerne les coûts d'utilisation et le manque d'autorisation aux accoucheuses de fournir les méthodes contraceptives à longue durée. Bien qu'au niveau national le protocole clinique général sur la PF et les outils de supervision existants soient suffisants, le protocole spécifique sur les soins postnatals ne donne pas beaucoup d'espace à la contraception. En tout cas, les résultats de cette étude montrent qu'il y a souvent un écart important entre les politiques et les protocoles, et leur application pratique dans l'offre de soins quotidien. 


\section{Recommandations (Objectif 5):}

Premièrement, des mesures doivent être prises pour améliorer la qualité de l'offre de services de PFPP. La présence d'agents de santé compétents et motivés qui sont autorisés et soutenus pour fournir des bons soins doit être garantie. À cet égard, les domaines d'action sont au niveau de formation pré-emploi et en cours d'emploi, ainsi que la supervision et soutien au personnel. Afin de pallier le manque de sages-femmes dans les zones rurales, il faudrait envisager de permettre aux accoucheuses d'être formés pour fournir les méthodes à longue durée, au moins comme stratégie de court terme. Le respect des protocoles cliniques devrait être amélioré, notamment en ce qui concerne le counseling et la fourniture d'informations. Dans des protocoles cliniques largement suffisants, des améliorations pourraient cependant être apportées. L'amélioration des compétences du personnel contribuera à rendre disponible une plus large gamme de méthodes contraceptives dans tout le pays. D'autres mesures sont aussi nécessaires pour éviter la récurrence des ruptures.

Deuxièmement, afin de faciliter l'accès et d'améliorer l'utilisation, il est nécessaire de faire un meilleur usage des possibilités existantes pour promouvoir la FP dans le continuum de soins. La mise en œuvre et l'amélioration du protocole sur les consultations prénatales (CPN) permettrait que la PF soit abordée déjà lors des soins prénatals, et d'autres mesures pourraient être prises pour promouvoir la préparation au postpartum et à la PFPP, à côté de la préparation à l'accouchement. L'élimination complète des paiements des utilisateurs en matière de contraception est nécessaire afin de faciliter l'accès, notamment pour les femmes les plus défavorisées.

Enfin, il est nécessaire de commencer à remettre en question la croyance répandue selon laquelle la culture traditionnelle est irrémédiablement hostile à la $\mathrm{PF}$, et de réaliser que les services de haute qualité ont le potentiel de stimuler la demande. À cet égard, fournir des conseils personnalisés et permettre un choix éclairé doivent devenir partie de la pratique courante. A cet effet, le développement d'une relation de confiance mutuelle entre prestataire et client est crucial. Promouvoir la participation des hommes a également le potentiel d'accroître la légitimité et promouvoir l'adoption de la PFPP.

STEP UP œuvre en faveur d'une recherche pertinente à l'élaboration des politiques afin de promouvoir une approche basée sur des données pour améliorer l'accès à la planification familiale et à l'avortement sans risques. Nous travaillons au Bangladesh, dans la partie Nord de l'Inde, au Ghana, au Kenya et au Sénégal.

Pour plus d'informations, veuillez contacter:

Dr. Harriet Birungi, hbirungi@popcouncil.org

Dr. lan Askew, laskew@popcouncil.org

\section{(C) 2014 LSHTM}

financé par

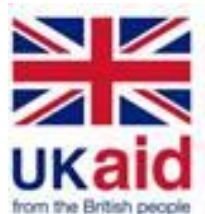

